\title{
BOLD responses to trigeminal nerve stimulation
}

\author{
Nathalie Just $^{\mathrm{a}, \mathrm{b}, *}$, Carl Petersen ${ }^{\mathrm{c}}$, Rolf Gruetter ${ }^{\mathrm{a}, \mathrm{b}, \mathrm{d}}$ \\ ${ }^{a}$ Laboratory for Functional and Metabolic Imaging, Ecole Polytechnique Fédérale de Lausanne, Lausanne, Switzerland \\ ${ }^{\mathrm{b}}$ Department of Radiology, University of Lausanne, 1015 Lausanne, Switzerland \\ ${ }^{\mathrm{c}}$ Laboratory of Sensory Processing, Brain Mind Institute, SV-BMI-LSENS AAB 105,Ecole Polytechnique Fédérale de Lausanne, 1015 Lausanne, Switzerland \\ ${ }^{\mathrm{d}}$ Department of Radiology, University of Geneva, 1205 Geneva, Switzerland \\ Received 7 September 2009; accepted 8 February 2010
}

\begin{abstract}
The current study investigates a new model of barrel cortex activation using stimulation of the infraorbital branch of the trigeminal nerve. A robust and reproducible activation of the rat barrel cortex was obtained following trigeminal nerve stimulation. Blood oxygen leveldependent (BOLD) effects were obtained in the primary somatosensory barrel cortex (S1BF), the secondary somatosensory cortex (S2) and the motor cortex. These cortical areas were reached from afferent pathways from the trigeminal ganglion, the trigeminal nuclei and thalamic nuclei from which neurons project their axons upon whisker stimulation. The maximum BOLD responses were obtained for a stimulus frequency of $1 \mathrm{~Hz}$, a stimulus pulse width of $100 \mu \mathrm{s}$ and for current intensities between 1.5 and $3 \mathrm{~mA}$. The BOLD response was nonlinear as a function of frequency and current intensity. Additionally, modeling BOLD responses in the rat barrel cortex from separate cerebral blood flow $(\mathrm{CBF})$ and cerebral metabolic rate of oxygen $\left(\mathrm{CMRO}_{2}\right)$ measurements showed good agreement with the shape and amplitude of measured BOLD responses as a function of stimulus frequency and will potentially allow to identify the sources of BOLD nonlinearities. Activation of the rat barrel cortex using trigeminal nerve stimulation will contribute to the interpretation of the BOLD signals from functional magnetic resonance imaging studies.
\end{abstract}

(C) 2010 Elsevier Inc. All rights reserved.

Keywords: Trigeminal nerve; Barrel cortex; BOLD; Neurovascular coupling; Modeling

\section{Introduction}

Because of the functional and morphological correlation between the vibrissae and the barrels, the whisker sensory system is an attractive model for studying structure, function, development and plasticity within the somatosensory cortex. The functional organization of the barrel cortex has a welldefined pathway between the whiskers and the cortex and allows the exploration of the link between molecular mechanisms, synaptic circuits and behavior (for a recent review see [1]). The signals travel by way of the infraorbital branch of the trigeminal nerve from the receptor cells in the whisker follicle to the trigeminal brainstem complex and eventually to the thalamus and the primary somatosensory cortex. In the thalamus, the ventral posterior medial nucleus

\footnotetext{
* Corresponding author. Laboratory for Functional and Metabolic Imaging (LIFMET), CIBM-EPFL, Batiment CH -Station 6, 1015 Lausanne, Switzerland. Tel.: +41 2169379 83; fax: +41 216937960 .

E-mail address: nathalie.just@epfl.ch (N. Just).
}

projects the majority of its axons to the barrels in layer IV of the somatosensory cortex which is arranged identically to the layout of the whiskers on the snout of the rodent. Following whisker stimulation, other cortical activations have been identified through cortico-cortical synaptic connections from primary to secondary somatosensory cortex and also from the somatosensory cortex to the motor cortex [1]. Contralateral connections have also been identified [1].

Sensory processing in the barrel cortex after deflection of one or more whiskers has been widely explored both in mice and rats [1-4]. Electrophysiological recordings [5] and optical imaging techniques have been developed through various experiments in order to obtain fast and spatially reliable functional mapping of the barrel cortex [6,7]. Intrinsic optical imaging has been used to map sensory processing in the mouse neocortex and relies on blood flow changes through coupling with neuronal activity [8]. This technique is widely used since it allows rapid and reliable measurements. Functional magnetic resonance imaging (fMRI) is a noninvasive method for mapping brain 
functional activity based on the blood oxygen leveldependent (BOLD) image contrast. BOLD contrast depends on localized decreases in cerebral deoxyhemoglobin (deox$\mathrm{yHb}$ ) content upon stimulation. While fMRI is widely used as an investigative tool to study human brain function [9], applications in rat brain are less frequent and most studies have focused on forepaw stimulation [10]. Only a handful studies have investigated whisker stimulation using fMRI [11-14]. The relationship between barrels and whiskers represents an interesting model to investigate neuronal activation, plasticity and neurovascular coupling. Nielsen and Lauritzen [15] used infraorbital nerve stimulation and found a non-linear relationship between cerebral blood flow (CBF) measured by laser Doppler flowmetry and the sum of local field potentials suggesting a nonlinear relationship between synaptic activity and CBF under specific experimental conditions $[15,16]$.

In the present work, we proposed to investigate the possibilities of obtaining optimal BOLD fMRI responses to electrical stimulation of the infraorbital branch of the trigeminal nerve in the rat barrel cortex at 9.4T. It is shown that (a) BOLD responses can be reliably detected in specific rat cortical areas such as the rat primary somatosensory barrel field cortex, (b) trigeminal nerve stimulation for barrel cortex activation is validated for fMRI studies in the rat and (c) BOLD responses can be deduced from $\mathrm{CBF}$ and cerebral metabolic rate of oxygen $\left(\mathrm{CMRO}_{2}\right)$ data and are in agreement with measured BOLD responses as a function of stimulus frequency both in magnitude and shape. The optimization and determination of optimal stimulation parameters of the trigeminal nerve stimulation model instead of the widely used forepaw stimulation model may allow collecting more information about the still unsolved question about neurovascular coupling.

\section{Materials and methods}

\subsection{Animal preparation}

All animal procedures were performed according to the federal guidelines of the Animal Care and approved by the local authority. Male adult Sprague-Dawley rats $(n=15$, 250-350 g) were initially anaesthetized with isoflurane in a mixture of $\mathrm{O}_{2}$. Each rat was orally intubated. A femoral artery and a femoral vein were catheterized for $\alpha$-chloralose administration and blood gas sampling. After fixing the rat head using ear and bite bars, the rat was positioned in a dedicated holder. The breathing rate was monitored simultaneously with body temperature throughout the experiment with a rectal probe (Model 1025, SA Instruments, Stony Brook, NY, USA). Body temperature was maintained at $37.5^{\circ} \mathrm{C} \pm 0.5^{\circ} \mathrm{C}$ with temperature-controlled circulating water placed under the rat. The blood pressure was monitored through a transducer attached to the cannulated femoral artery (SA Instruments).

Blood gases were sampled every $30 \mathrm{~min}$, and blood parameters were maintained at physiological levels
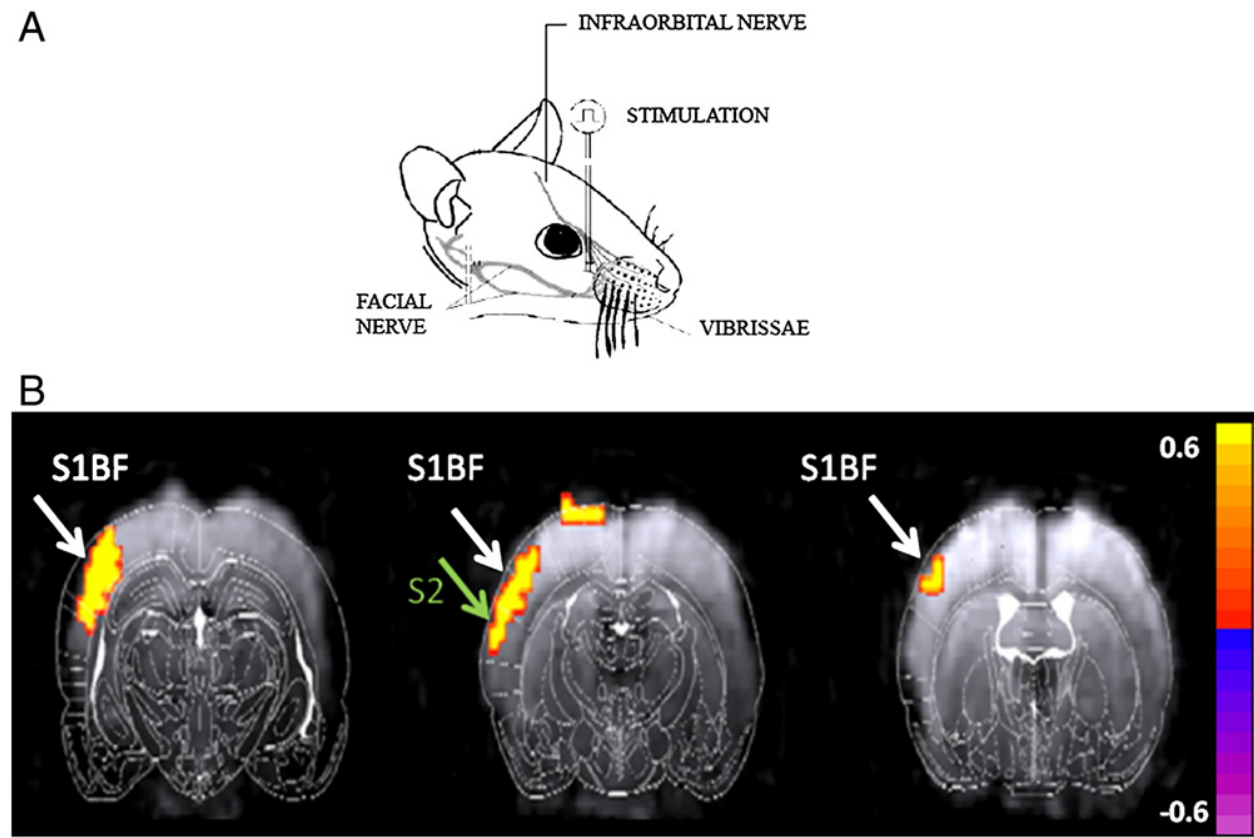

Fig. 1. A. Diagram showing the location of the infraorbital nerve and the position of electrodes for trigeminal nerve stimulation. B. Example of cross-correlation map showing activation of the rat cortex following trigeminal nerve stimulation and overlaid over a T2*-weighted single-shot gradient echo EPI image $(\mathrm{TE}=25 \mathrm{~ms})$. BOLD responses of one animal in 3 consecutive slices are displayed as cross-correlation maps. T-positive (red/yellow) BOLD responses were observed (color bar displaying maximum cross-correlation factors: 0.6 for positive BOLD and -06 for negative BOLD). The Paxinos and Watson atlas [32] corresponding to the slice of interest was fitted to images. The primary somatosensory barrel cortex field (S1BF) is localized between 0.26 and 4.16 mm posterior from the bregma [32]. The white and green arrows show activation of the primary somatosensory barrel cortex (S1BF) and the secondary somatosensory cortex (S2) respectively. 
$(\mathrm{pH}=7.35-7.4, \mathrm{pCO} 2=39-45 \mathrm{mmHg}$ and mean arterial blood pressure $=90-130 \mathrm{mmHg}$ ) throughout the experiment.

After surgery, anesthesia was switched from isoflurane to $\alpha$-chloralose; an initial intravenous dose of $80 \mathrm{mg} / \mathrm{kg}$ was administered followed by a continuous intravenous infusion of $27 \mathrm{mg} / \mathrm{kg}$ per hour at a rate of $2 \mathrm{ml} / \mathrm{h}$.

\subsection{Trigeminal nerve stimulation}

A few experimental sessions were dedicated to the accurate localization of the infraorbital nerve. An anterior-posterior skin incision was made $2 \mathrm{~mm}$ above the left eye following the frontal bone. The skin, muscle and orbital content were retracted laterally to expose the edge of the frontal bone and the infraorbital nerve (ION) respectively. The ION was exposed downed to the eye corner where it lies approximately 1-2 $\mathrm{mm}$ deep (hiatus infraorbitalis) (Fig. 1A).

For further studies and to avoid damaging other nerves and creating further susceptibility effects, experiments were then performed without surgery. Two stainless steel electrodes were percutaneously inserted either in the left or right trigeminal nerve. The cathode was inserted in the hiatus infraorbitalis as described in [15] and the anode was inserted either in the masticatory muscles or the neck muscles. Electrical stimulation of one trigeminal nerve was performed by delivering square pulses using an external stimulator (WPI, Stevenage, UK). Within one fMRI experiment, all the stimulus parameters were kept constant (Current, pulse width and frequency). The paradigm used was $60 \mathrm{~s}$ OFF and $30 \mathrm{~s}$ ON repeated during 10-12 min.

fMRI All the experiments were performed on an actively shielded $9.4 \mathrm{~T} / 31 \mathrm{~cm}$ bore magnet (Magnex, Varian, Abingdon, UK) with $12 \mathrm{~cm}$ gradients ( $400 \mathrm{mT} / \mathrm{m}$ in $120 \mu \mathrm{s})$ with a quadrature transmit/receive $17 \mathrm{~mm}$ surface coil. First and second order shims were adjusted using FASTM AP [17] resulting in water linewidths of $13-15 \mathrm{~Hz}$ in a $216-\mu 1$ volume. The BOLD response was assessed using single shot gradient echo echo planar imaging (EPI) $(\mathrm{TR} / \mathrm{TE}=2500-2000 / 25 \mathrm{~ms}$; FOV $=20 \times 20 \mathrm{~mm}$; matrix $=64 \times 64$; slice thickness $=1 \mathrm{~mm} ; 10$ slices, bandwidth $=325 \mathrm{~K} \mathrm{~Hz}, 300$ volumes). The echo realignment was performed using a reference scan [18].

In a first set of experiments $(n=8)$, the following frequencies were used: $0.25,0.5,1.0,2.0,3.0,5,8,10$ and $12 \mathrm{~Hz}$ with a pulse duration of $100 \mu \mathrm{s}$ and a current amplitude of $2 \mathrm{~mA}$.
In a second set of experiments $(n=13)$, currents were varied from 1 up to $6 \mathrm{~mA}$ with pulse duration of $100 \mu \mathrm{s}$ at a frequency of $1 \mathrm{~Hz}$.

A rest period of 10-12 min was introduced before increasing the pulse width, stimulus frequency or current intensity.

Data Analysis Data were processed using STIMULATE (University of Minnesota, Minneapolis, MN, USA) [19] and MATLAB routines (The MathWorks, Natick, MA, USA). Stimulation induced activation $t$ value maps, and crosscorrelation maps were calculated on a pixel by pixel basis from the comparison between the experimental fMRI data acquired during the control periods and the stimulation periods and the cross-correlation of the motion-corrected and $3 \times 3 \times 3$ Gaussian smoothed time series with a boxcar waveform representing the stimulation period, respectively. The minimal cross-correlation activation threshold was set to 0.3 . Only clusters comprising at least five pixels were considered significant $(P<.05)$. Regions of interest (ROIs) encompassing the activated primary somatosensory barrel field cortex (S1BF) were drawn. ROIs were on average were $16 \pm 10$ pixels. An average time-course was recorded for each animal. For each rat and each condition investigated, six to eight successive BOLD responses were averaged per time course. No baseline correction was performed.

Cross-correlation maps were overlaid on single shot gradient echo EPI images [18]. Data are presented as means \pm S.E.M. Mean BOLD responses were defined as averages between 25 and $55 \mathrm{~s}$ during the stimulation period. One-way analysis of variance (ANOVA) tests were performed to assess significance between means of BOLD responses. $P<.05$ was considered significant.

\subsection{Modeling $\mathrm{BOLD}$ responses from $\mathrm{CBF}$ and $\mathrm{CMRO}_{2}$ data in the barrel cortex}

In order to model the BOLD response in the barrel cortex based on $\mathrm{CBF}$ and $\mathrm{CMRO}_{2}$ data, the following equation was used [20,21]:

$\frac{\Delta \mathrm{BOLD}}{\mathrm{BOLD}}=M\left(1-\left(\frac{C M R O 2}{C M R O 20}\right)^{\beta}\left(\frac{C B F}{C B F 0}\right)^{\alpha-\beta}\right)$

$M$ is a proportionality constant; the parameters with subscripts 0 indicate baseline values. $\alpha$ and $\beta$ reflect the

Table 1

Cross-laboratory comparisons of baseline $\mathrm{CBF}$ values

\begin{tabular}{|c|c|c|c|c|}
\hline $\mathrm{CBF}_{0}{ }^{\mathrm{a}}$ & Anaesthesia & Magnetic field strength & Brain regions & Reference \\
\hline $0.4 \pm 0.9$ & Morphine & $7 \mathrm{~T}$ & Rat somatosensory cortex & Hyder et al. [24] \\
\hline $0.69 \pm 0.17<0.41-0.95>^{b}$ & $\alpha$-chloralose & $9.4 \mathrm{~T}$ & Rat somatosensory cortex & Duong et al. [25] \\
\hline $0.72 \pm 0.19$ & $\alpha$-Chloralose & Autoradiography & Rat somatosensory cortex & Ueki et al. [26] \\
\hline $0.91 \pm 0.31$ & Kétamine & $9.4 \mathrm{~T}$ & & \\
\hline
\end{tabular}

${ }^{\mathrm{a}}$ All values are in $\mathrm{ml.g}{ }^{-1} \mathrm{~min}^{-1}$.

b Range of values Ref. [25]. 
Table 2

Cross-laboratory comparisons of $M$ values

\begin{tabular}{llll}
\hline$M$ & Anaesthesia & Magnetic field strength & Brain regions \\
\hline $0.05 \pm 0.01$ & Isoflurane & $4.7 \mathrm{~T}$ & Rat Forepaw somatosensory cortex \\
$0.079 \pm 0.007<0.053-0.12>^{\mathrm{a}}$ & None & $1.5 \mathrm{~T}$ & Humans - primary visual cortex et al. [28] \\
$0.16 \pm 0.02$ & Urethane & $3 \mathrm{~T}$ & Rat Brain Davis et al. [20] \\
$0.15 \pm 0.06-0.22 \pm 0.06$ & None & $1.5 \mathrm{~T}$ & Human visual cortex \\
$0.12 \pm 0.03$ & Isoflurane & $4.7 \mathrm{~T}$ & Rat Forepaw somatosensory cortex \\
\hline
\end{tabular}

a Range of values given in Ref. [20].

effect of blood volume and deoxyhemoglobin concentration to the BOLD signals, respectively. $\alpha=.29$ [22] and $\beta=1.5$ [20] were used. We used $\mathrm{CMRO}_{20}=256.9 \pm 11.5 \mu \mathrm{mol} / 100 \mathrm{~g} /$ min [23] and $\mathrm{CBF}_{0}=0.4,0.69$ and $0.91 \mathrm{ml} / \mathrm{g}$ per minute (Table 1). $M$ reflects the baseline deoxyHb content and is also defined as the maximum BOLD change for the baseline state in the region of interest. M values were $0.05,0.079$ and 0.16 (Table 2) for the modeling procedure.

$\mathrm{CBF}$ and $\mathrm{CMRO}_{2}$ changes as a function of stimulus frequency measured in the rat barrel cortex following trigeminal nerve stimulation as described in [23] were used to model BOLD responses.

\section{Results}

As depicted in Fig. 1B, activation was detected on three to six consecutive slices in an area corresponding to the barrel cortex of the rat following trigeminal nerve stimulation and while varying stimulus frequency and current intensity. No changes in BOLD were found while varying the stimulus pulse width from $50 \mu$ s to $10 \mathrm{~ms}$. For further experiments, a pulse width of $100 \mu \mathrm{s}$ was chosen at it demonstrated the most stable BOLD response during 30-s stimulation.

\subsection{Effect of stimulus frequency}

Strong and robust activation was detected in the primary somatosensory barrel field cortex (S1BF) in all the rats when the stimulus frequency was above $0.5 \mathrm{~Hz}$ (Fig. 1B, Fig. 2A). Activation was also detected in the secondary somatosensory cortex (S2) at $0.5(n=5), 1(n=7), 2(n=6), 3(n=7)$ and $5 \mathrm{~Hz}$ $(n=4)$ (Fig. 2A) and in the motor cortex at 1,2 and $3 \mathrm{~Hz}$ and up to $12 \mathrm{~Hz}$ in all the rats. The mean temporal profiles of the BOLD responses showed reproducible 30 -s responses at stimulus frequencies of 0.5 and $1 \mathrm{~Hz}$ (Fig. 2B). At stimulus frequencies of 0.25 and $2 \mathrm{~Hz}$, the BOLD response decreased during the stimulation period. At $3 \mathrm{~Hz}$, an overshoot after stimulus onset was seen in each rat of the study $(n=8)$. At 1 and $2 \mathrm{~Hz}$, a poststimulus BOLD undershoot was observed in each animal.

Fig. $2 \mathrm{C}$ shows that the magnitude of the BOLD response varied as a function of stimulus frequency. The mean BOLD response reached a maximum $(4.6 \pm 0.5 \%, n=7)$ for a stimulus frequency of $1 \mathrm{~Hz}$. At $0.5(3.4 \pm 0.5 \%), 1(4.6 \pm 0.5 \%)$ and 2 (3.4 $\pm 0.5 \%, n=7)$, no significant differences were found between the BOLD amplitudes (ANOVA, $P>.05$ ). At $1 \mathrm{~Hz}$, BOLD amplitudes were significantly higher than at 0.25 and
$3 \mathrm{~Hz}$ (ANOVA: $P=.0008$ and $P=.009$ respectively) but not than at 0.5 and $2 \mathrm{~Hz}$ (ANOVA, $P>.05$ ). Above $1 \mathrm{~Hz}$ and up to $12 \mathrm{~Hz}$, the BOLD responses declined continuously to a magnitude of $1.37 \% \pm 0.8 \%$ at $12 \mathrm{~Hz}$.

\subsection{Effect of stimulation intensity}

Based on the robustness, reproducibility and BOLD amplitude responses, the stimulus frequency was fixed at 1 $\mathrm{Hz}$, while the current intensity was varied from 0.75 to $6 \mathrm{~mA}$. Blood pressure was not affected while increasing the stimulation strength up to a current intensity of $3 \mathrm{~mA}$. Above $3 \mathrm{~mA}$, the blood pressure dropped (from 100-120 to $80 \mathrm{mmHg}, n=6$ ), and significant fluctuations in the BOLD amplitudes were observed between rats: (between 4 and 6 $\mathrm{mA}$, the BOLD amplitude response varied between 3.4 and $9.6 \% ; n=6)$.

The activation extended to the secondary somatosensory cortex (S2) in all the rats at current intensities above $1.3 \mathrm{~mA}$ and to the motor cortex in all the rats for current intensities above $2 \mathrm{~mA}$. Activation was also detected in the contralateral secondary somatosensory cortex.

The temporal profile of the BOLD response showed a tendency to decrease during the course of stimulation (Fig. 3A). However, the average BOLD response increased as a function of current intensity up to $1.3 \mathrm{~mA}$. Significant differences were found between BOLD responses at 0.75 and $1 \mathrm{~mA}$ (ANOVA test, $P<.01$ ) and between BOLD responses at 1 and $1.3 \mathrm{~mA}$ (ANOVA test, $P<.00001$ ). Above $1.3 \mathrm{~mA}$, the BOLD response plateaued and no significant differences were observed (ANOVA test, $P>.05$ ).

Fig. $3 \mathrm{~B}$ shows the mean BOLD percentage change as a function of current intensity up to an intensity of $3 \mathrm{~mA}$. Below $0.75 \mathrm{~mA}$, discriminating between BOLD responses and signal fluctuations became difficult leading to low BOLD responses of $0.15 \pm 0.2 \%$. From 0.75 to $1.3 \mathrm{~mA}$, the mean BOLD signal amplitude increased from $1.3 \%$ to $2.6 \%$ (ANOVA, $P=.00024$ ) demonstrating a threshold above 1 $\mathrm{mA}$. From 2 to $3 \mathrm{~mA}$, the BOLD amplitude remained constant with mean amplitude $2.4 \pm 0.1 \%$.

The BOLD response as a function of current intensity was characterized by an early decrease in BOLD responses that peaked around 2-2.5 $\mathrm{s}$ after the onset of trigeminal nerve stimulation followed by a BOLD increase reaching a maximum in 8-10 $\mathrm{s}$ post stimulation onset for current intensities above $1 \mathrm{~mA}$. The magnitude of this pre-BOLD 

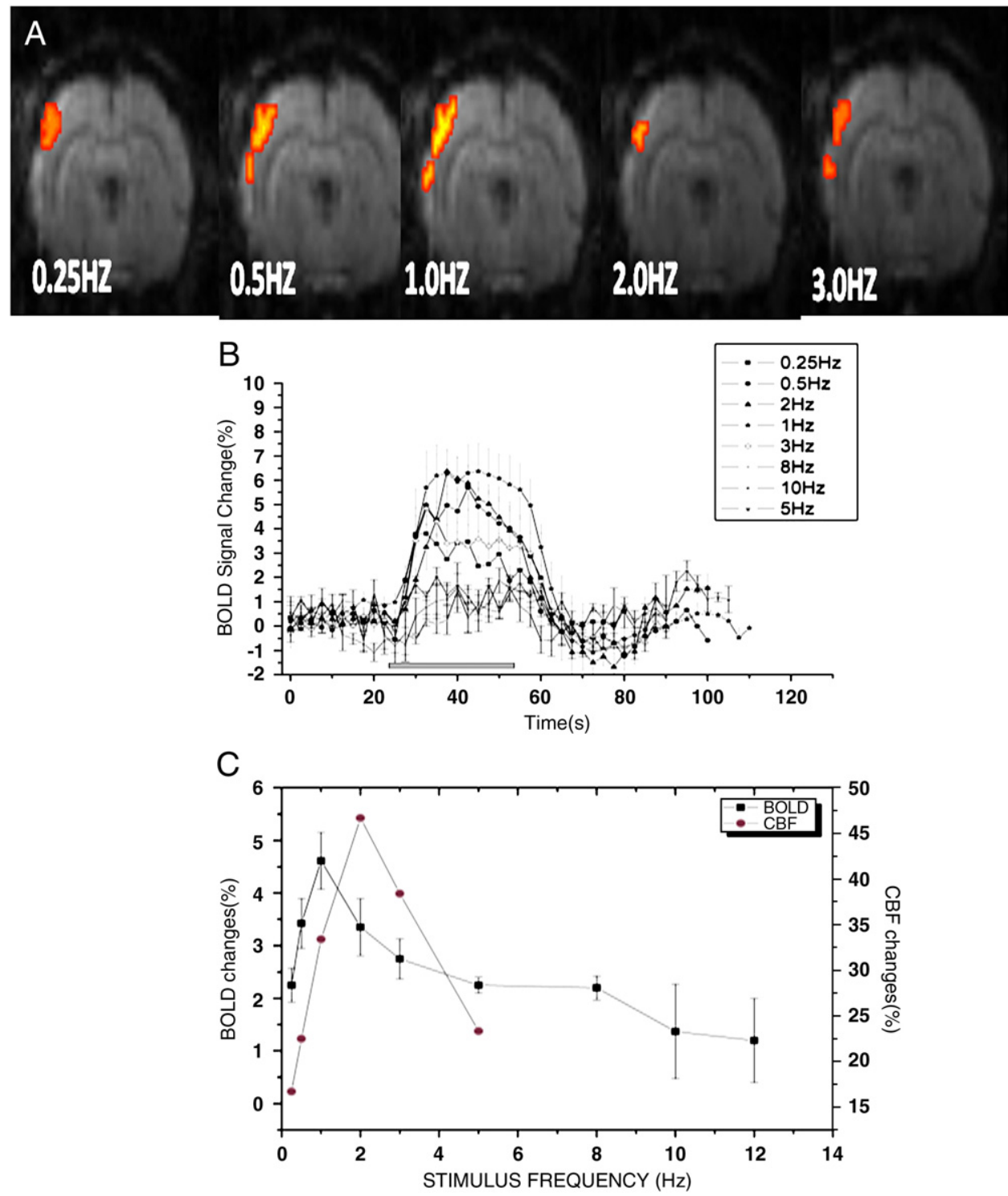

Fig. 2. Stimulus frequency dependence of barrel cortex BOLD response in $\alpha$-chloralose anesthetized rats. (A) Cross-correlation maps overlaid over single shot gradient-echo EPI images (TE=25 ms) of the same slice in the same rat and at $0.25,0.5,1,2$ and $3 \mathrm{~Hz}$. These images were all identically scaled and show activation in S1BF at all stimulation frequencies as well as in S2 at 0.5, 1 and $3 \mathrm{~Hz}$. (B) Average time courses within S1BF of 8 animals are plotted for frequencies $0.25,0.5,1,2,3,5$ and $10 \mathrm{~Hz}$ (Mean \pm S.E.M, $n=8$ ). The grey bar under the time courses indicates the 30 -s stimulation period. (C) BOLD response amplitude versus stimulus frequency in S1BF. For comparison purposes, the mean CBF changes obtained in Nielsen et al. [15] were also represented as a function of stimulus frequency.

decrease or "initial dip" was more pronounced for current intensities above $2 \mathrm{~mA}$. The initial dip was consistently detected among 13 animals at all current intensities above $1.3 \mathrm{~mA}$ (Fig. 3C). Between 0.75 and $1 \mathrm{~mA}$, the "initial dip" was more difficult to detect and could only be seen in $2-3$ rats.

\subsection{Comparison between measured BOLD responses and modeled BOLD responses in the rat barrel cortex}

Fig. 4A presents the modeled BOLD responses as a function of stimulus frequency up to $40 \mathrm{~Hz}$ under three gradually increasing CBF baseline values taken from the literature (Table 1) and for an assumed $M=0.079$. Modeled
BOLD responses as a function of stimulus frequency were non-linear and demonstrated increased magnitudes as $\mathrm{CBF}_{0}$ decreased. Maximum modeled BOLD responses were $5.23 \%, 4 \%$ and $3.3 \%$ for baseline $\mathrm{CBF}$ values of $0.4,0.69$ and $0.91 \mathrm{ml} \cdot \mathrm{g}^{-} 1 \mathrm{~min}^{-1}$, respectively. The measured BOLD responses demonstrated a sharpest evolution as a function of stimulus frequency compared to the modeled BOLD and a fast decline for frequencies above $1 \mathrm{~Hz}$.

As $M$ was increased, the BOLD magnitude was also significantly increased ranging from 3.3\%, 5.2\% and $10.6 \%$ at $1 \mathrm{~Hz}$ and $\mathrm{CBF}_{0}=0.4 \mathrm{ml} / \mathrm{g}$ per minute. For each given $M$ value, BOLD amplitudes were increased as baseline CBF decreased (Fig. 4B). 

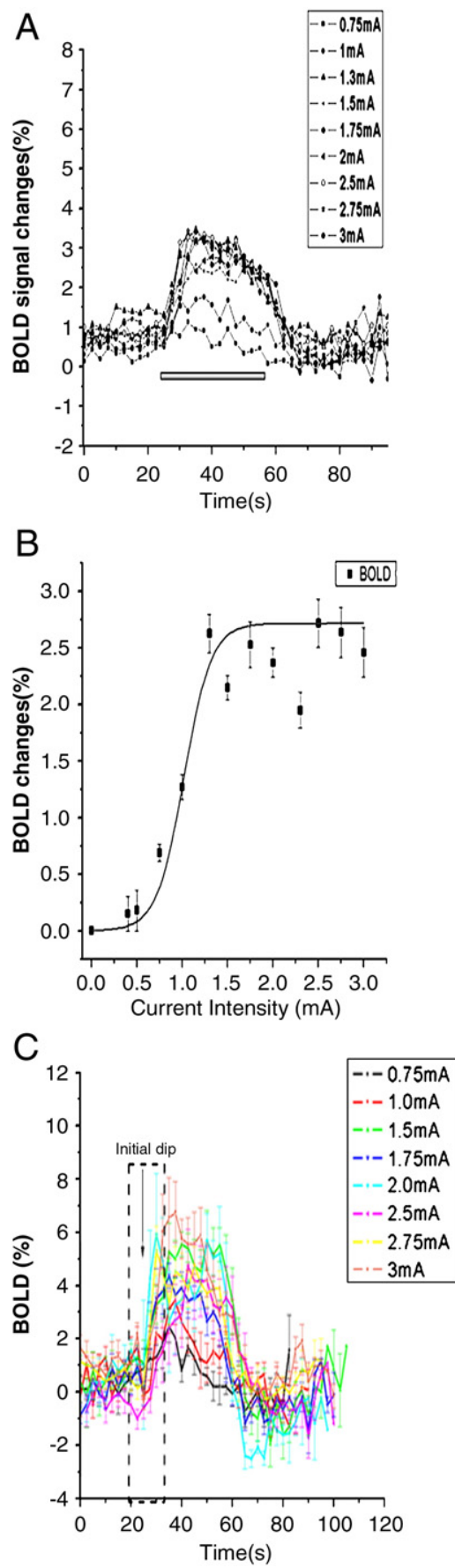

Fig. 3. Stimulus current intensity dependence of barrel cortex BOLD response in $\alpha$-chloralose anesthetized rats. (A) Average time courses within S1BF are plotted successively for $0.75-1.3 \mathrm{~mA}, 1.5-2 \mathrm{~mA}, 2.5-3 \mathrm{~mA}$. The grey bar under the time courses indicates the 30 -s stimulation period. (B) BOLD response amplitude versus current intensity in the primary somatosensory barrel cortex (S1BF) (mean \pm S.E.M. $(n=13)$. Experimental points were fitted using a sigmoidal model (represented as the smooth curve) to show the nonlinear behavior of BOLD responses as a function of stimulus strength. From 0.75 to $1.5 \mathrm{~mA}$, the mean BOLD signal amplitude increased from $1.3 \%$ to $2.6 \%$ (ANOVA, $P<.01$ ). From 1.3 to $3 \mathrm{~mA}$, the BOLD amplitude remained almost constant with an average amplitude of $2.4 \pm 0.1$ \%. (C) Example of BOLD responses time courses in a single animal as a function of current intensity and demonstrating a BOLD decrease or "initial dip" post-stimulation onset.
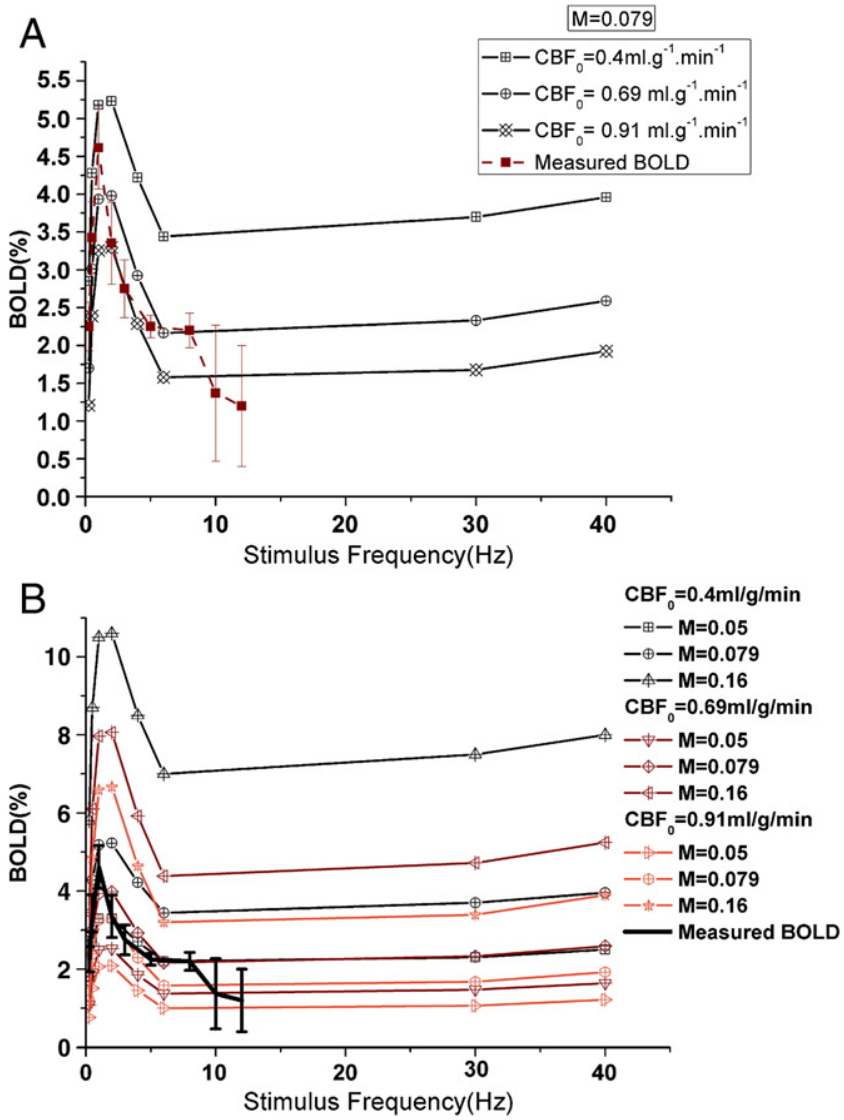

Fig. 4. Comparison of modeled and measured BOLD responses in the rat barrel cortex. Modeled BOLD responses from $\mathrm{CBF}$ and $\mathrm{CMRO}_{2}$ values in the rat barrel cortex following trigeminal nerve stimulation [23] for A $M=0.079$ and $\mathrm{CBF}_{0}=0.4,0.69$ and $0.91 \mathrm{ml.g} \mathrm{g}^{-1} \mathrm{~min}^{-1}$ and for B. $M=0.05$, 0.079 and 0.16 successively modeled with $\mathrm{CBF}_{0}=0.4,0.69$ and $0.91 \mathrm{ml} . \mathrm{g}^{-} 1$ $\min ^{-1}$. The measured BOLD responses are plotted for comparison.

The largest measured BOLD response was $4.6 \% \pm 0.5 \%$ $(1 \mathrm{~Hz})$ while the smallest was $1.37 \% \pm 0.8 \%(12 \mathrm{~Hz})$. BOLD estimates using Eq. (1) were closest to experimental findings for $M=0.079$ independently of the baseline CBF values.

\section{Discussion}

\subsection{Localization of BOLD activation following trigeminal nerve stimulation}

The BOLD response upon trigeminal nerve stimulation using electrodes and under $\alpha$-chloralose anesthesia was measured as a function of stimulus frequency and current intensity. We found robust and reproducible BOLD responses in the barrel field primary somatosensory cortex (S1BF). In addition to evoking responses in S1BF, we also found prominent evoked responses in $\mathrm{S} 2$ and motor cortex in excellent agreement with a previous voltage-sensitive dye imaging study [7] and an EEG imaging study [31].

The locations of the center of the BOLD activation maps were in excellent agreement with the coordinates of the barrel cortex, the secondary somatosensory cortex and the 
motor cortex areas [32]. These activations resulted from the afferent signals transported from the trigeminal nuclei to the thalamus and finally to the barrel field of the somatosensory cortex in agreement with the established pathways of the whisker sensory system $[7,33]$.

\subsection{Nonlinearities of the BOLD response to trigeminal nerve stimulation}

The understanding of the physiological basis of the BOLD contrast and in particular, the relationship between neural activity and the hemodynamic response are important for an appropriate interpretation of functional imaging signals. Several recent studies evaluated the effect of stimulus frequency and current intensity on neuronal activity and BOLD under different anesthesia conditions using forepaw stimulation [10,34,35] and the more complex whisker stimulation [36]. In our study, the BOLD response increased linearly as a function of frequency up to $1 \mathrm{~Hz}$ and decreased for stimulus frequencies up to $12 \mathrm{~Hz}$ demonstrating an overall nonlinear response.

For stimulation frequencies above $5 \mathrm{~Hz}$, barrel cortex BOLD responses were not reproducibly detected in all rats. In $\alpha$-chloralose studies, the highest stimulus frequencies for which BOLD signal changes were still detected ranged between 3 and $5 \mathrm{~Hz}[15,35,37,38]$. However, it is likely that the type of anesthesia may affect the relationship between the neural activity and hemodynamic response. Linear coupling between neural and hemodynamic responses (CBF) was observed up to a frequency of $40 \mathrm{~Hz}$ in conscious animals undergoing electrical stimulation of the whisker pad [39], whereas uncoupling was shown above $5 \mathrm{~Hz}$ in urethaneanesthetized rats. These results showed that anesthesia may affect the mechanism of coupling of CBF and BOLD to functional neural activity. Maximal neuronal and hemodynamic responses were obtained at low frequency stimulation under $\alpha$-chloralose anesthesia $[15,35]$. Under $\alpha$-chloralose anesthesia, decreases in neural and hemodynamic activities have been attributed to increases in synaptic inhibition or deactivation of glutamatergic transmission [35].Therefore, the decrease in neuronal activity and BOLD response at frequencies higher than $5 \mathrm{~Hz}$ for forepaw stimulation and 2 $\mathrm{Hz}$ for trigeminal stimulation may be related to increased synaptic inhibition in the primary somatosensory cortex caused by decreased activity in a projecting brain region such as the thalamus.

$\alpha$-Chloralose anesthesia is known to be a minimal depressant of neural functions [40]. Moreover, it was shown that $\alpha$-chloralose anesthesia during functional activation showed similar patterns of metabolic activation in the primary somatosensory cortex of rats to that in conscious animals [26]. Nonetheless, Nakao et al. [41] showed that $\alpha$-chloralose reduced local cerebral blood flow and glucose utilization in the barrel cortex as well as in the thalamic ventral posterior medial nucleus. Reduced cerebral blood flow due to $\alpha$-chloralose anesthesia may therefore explain why we did not detect activation in the thalamus as would be expected from the established pathways to the barrel cortex [32]. In addition, the pattern of activation may be spatially frequency-dependent $[23,42]$. Therefore thalamic activation may not be detectable at the frequencies used in the present study. This result is interesting for further connectivity studies using the model of trigeminal nerve stimulation.

\subsection{Neurovascular coupling in the rat barrel cortex}

A linear correlation between neuronal activity and BOLD response both measured with various methods was suggested by a large number of studies [43-47]. In some cases, this proportionality between neuronal activity and hemodynamic response was restricted to specific conditions $[15,48]$ with several studies demonstrating nonlinear effects [49].

In the present work, a sigmoïdal behavior of the BOLD response was found between $0.4 \mathrm{~mA}$ and $3 \mathrm{~mA}$ as a function of current intensity and demonstrated a BOLD threshold above a current intensity of $1 \mathrm{~mA}$. Nielsen and Lauritzen [15] also suggested, a $\mathrm{CBF}$ threshold since no $\mathrm{CBF}$ changes were detected at low current intensities $(0-0.5 \mathrm{~mA})$ but neuronal activity was still present with low field potential (FP) amplitudes up to $2.0-2.5 \mathrm{mV}$. If the neuronal activity must reach a threshold before any BOLD can be detected, it would imply that a threshold level for energy demand or release of neurotransmitters is needed before the neurovascular signaling results in a hemodynamic response [42,49]. Following trigeminal nerve stimulation, $\mathrm{CMRO}_{2}$ changes as a function of field potentials were constant as a function of low field potentials [23] while small CBF and FP increases were detected. This result indicates that other mechanisms such as glycolysis or mitochondrial oxygen buffer [50] may be involved during low neural activity until a threshold is reached triggering a decrease in the oxygen extraction fraction and an increased hemodynamic response. The "threshold model" remains a controversial issue as the sensitivity of BOLD and $\mathrm{CBF}$ techniques are still questioned for small changes in neural activities [49]. Based on the present study and the CBF measurements using laser Doppler flowmetry which demonstrated that a threshold was needed for CBF changes to be detected, we propose that a physiological threshold is necessary to elicit a hemodynamic response.

The "initial dip" was defined as an initial deoxygenation peak attributed to an early focal increase in oxygen consumption triggered by an increase of oxygen extraction fraction followed by flow increase [50]. The presence of an initial dip was found in the whisker barrel cortex for current intensities above $1 \mathrm{~mA}$ following Jones's findings [22] but opposing Lindauer et al. [40] who did not find evidence of an early deoxyhemoglobin increase during barrel cortex activation. This issue is of particular interest as the initial dip presence may allow in the future a more spatially accurate activation mapping. The initial dip may be more localized and specific to activated barrels in the layers of the 
barrel cortex than the later BOLD response [50]. It may also help understand nonlinearities since it provides means to probe the link between oxygen consumption and hemodynamic response.

\subsection{Modeling of the BOLD effect}

The BOLD effect can be estimated using either models of intravascular susceptibility differences $[51,52]$ or models of $\mathrm{CBF}, \mathrm{CBV}, \mathrm{CMRO}_{2}$ changes during activation $[20,52]$. In the present study, the measured BOLD during trigeminal nerve stimulation was compared to the modeled BOLD using Davis's model [20] and CBF and $\mathrm{CMRO}_{2}$ measurements performed with the same paradigm of stimulation [23]. Although a number of assumptions were made regarding $\mathrm{M}$, $\alpha$ and baseline $\mathrm{CBF}$ values (Tables 1 and 2) and although BOLD, $\mathrm{CBF}$ and $\mathrm{CMRO}_{2}$ measurements were separate, measured and modeled BOLD responses as a function of stimulus frequency were in agreement in both magnitude and shape. Moreover, the modeling process showed evidence that baseline CBF can have a strong effect on the magnitude of the BOLD response to the same stimulus. As $\mathrm{CBF}_{0}$ was increased modeled BOLD amplitudes decreased showing agreement with the measured BOLD for $\mathrm{M}=0.079$. M plays an important role in the modeling which was accurately described in several studies [20,52]. As M represents the baseline deoxyHb concentration scaled in terms of BOLD signal which is dependent on the washout of this deoxyHb, we may hypothesize that $\mathrm{M}$ as well as $\mathrm{CBF}_{0}$ vary between changes in stimulus frequency although differences between measured and modeled BOLD responses may be attributed to experimental changes between BOLD and $\mathrm{CBF}$ and $\mathrm{CMRO}_{2}$ measurements.

In the present study, we showed that the combination of non-linear $\mathrm{CBF}$ changes and linear $\mathrm{CMRO}_{2}$ changes both as a function of stimulus frequency resulted in non-linear BOLD estimates as a function of stimulus frequency in the rat barrel cortex in accordance with predictions by Buxton et al. [52] suggesting that a large part of the non-linearity of the BOLD response arises from the CBF response. This method provides a way to investigate the sources of nonlinearities in BOLD responses.

In conclusion, stimulation of trigeminal nerve can be used to investigate the mechanism of vascular coupling as well as the organization of the whisker sensory system using fMRI.

\section{Acknowledgments}

Supported by the centre d'Imagerie Médicale (CIBM) of UNIL, EPFL, HUG, CHUV and the Leenards and Jeantet Foundations. We thank Dr Martin Lauritzen for useful contributions to this manuscript.

\section{References}

[1] Petersen $\mathrm{CCH}$. The functional organization of the barrel cortex. Neuron 2007;56:339-55.
[2] Petersen $\mathrm{CCH}$, Sakmann B. The excitatory neural network of rat layer 4 barrel cortex. J Neurosci 2000;20:7579-86.

[3] Brecht M. Barrel Cortex and whisker-mediated behaviors. Curr Opin Neurobiol 2007;17:408-16.

[4] Kleinfeld D, Ahissar E, Diamond ME. Active Sensation: Insight from the rodent vibrissa sensorimotor system. Curr Opin Neurobiol 2006;16:435-44.

[5] Petersen RS, Panzeri S, Diamond ME. Population coding of stimulus location in the rat somatosensory cortex. Neuron 2001;32:503-14.

[6] Ferezou I, Bolea S, Petersen CC. Visualizing the cortical representation of whisker touch: voltage-sensitive dye imaging in freely moving mice. Neuron 2006;18:617-29.

[7] Ferezou I, Haiss F, Gentet LJ, Aronoff R, Weber B, Petersen CCH. Spatiotemporal dynamics of cortical sensorimotor integration in behaving mice. Neuron 2007;56:907-23.

[8] Vanzetta I, Grinvald A. Coupling between neuronal activity and microcirculation: implications for functional brain imaging. HFSP J 2008;2:79-98.

[9] Turner R, Howseman A, Rees GE, Josephs O, Friston K. Functional magnetic resonance imaging of the human brain: data acquisition and analysis. Exp Brain Res 1998;123:5-12.

[10] Van Camp N, Verhoye M, Van der Linden A. Stimulation of the rat somatosensory cortex at different frequencies and pulse widths. NMR Biomed 2006;19:10-7.

[11] Lu H, Mazaheri Y, Zhang R, Jesmanowicz A, Hyde JS. Multishot partial k-space EPI for high -resolution fMRI demonstrated in a rat whisker barrel stimulation model at 3T. Magn Reson Med 2003;50: $1215-22$.

[12] Alonso de CB, Lowe AS, Dear JP, Lee KC, Williams SC, Finnerty GT. Sensory inputs from whisking movements modify cortical whisker maps visualized with functional magnetic resonance imaging. Cereb Cortex 2008;18:1314-25.

[13] Yang X, Hyder F, Shulman RG. Activation of a single whisker barrel in rat brain localized by functional magnetic resonance imaging. Proc Natl Acad Sci U S A 1996;96:475-8.

[14] Yang X, Hyder F, Shulman RG. Functional MRI BOLD signal coincides with electrical activity in the rat whisker barrels. Magn Reson Med 1997;38:874-7.

[15] Nielsen AN, Lauritzen M. Coupling and uncoupling of activitydependent increases of neuronal activity and blood flow in rat somatosensory cortex. J Physiol 2001;533:773-85.

[16] Lauritzen M, Gold L. Brain function and neurophysiological correlates of signals used im functional neuroimaging. J Neurosci 2003;23: 3972-80.

[17] Mlynarik V, Gambarota G, Frenkel H, Gruetter R. Localised short echo time MR spectroscopy with full signal-intensity acquisition. Magn Reson Med 2006;56:965-70.

[18] Lei H, Mlynarik V, Just N, Gruetter R. Snapshot gradient-recalled echo-planar images of rat brain at long echo time at $94 \mathrm{~T}$. Magn Reson Imaging 2008;26:954-60.

[19] Strupp JP. Stimulate a GUI based fMRI analysis software package. Neuroimage 1996;3:S607.

[20] Davis TL, Kwong KK, Weisskoff RB, Rosen B. Calibrated functional MRI: mapping the dynamics of oxidative metabolism. Proc Natl Acad Sci U S A 1998;95:1835-9.

[21] Hoge RD, Atkinson J, Gill B, Crelier GR, Marrett S, Pike GB. Investigation of BOLD signal dependence on cerebral blood flow and oxygen consumption: the deoxyhemoglobin dilution model. Magn Reson Med 1999;42:849-63.

[22] Jones M, Berwick J, Johnston D, Mayhew J. Concurrent optical imaging spectroscopy: the relationship between blood flow oxygenation and volume in rodent barrel cortex. Neuroimage 2001;13: $1002-15$.

[23] Enager P, Piilgaard H, Offenhauser N, Kocharyan A, Fernandes P, Hamel E, et al. Pathway-specific variations in neurovascular and neurometabolic coupling in rat primary somatosensory cortex. J Cereb Blood Flow Metab 2009;29:976-86. 
[24] Hyder F, Kennan RP, Kida I, Mason G, F Behar KL, Rothman D. Dependence of oxygen delivery on blood flow in rat brain: a 7 Tesla nuclear magnetic resonance study. J Cereb Blood Flow Metab 2000;20:485-98.

[25] Duong TQ, Silva AC, Lee SP, Kim SG. Functional MRI of calciumdependent synaptic activity: cross correlation with CBF and BOLD measurements. Magn Reson Med 2000;43:383-92.

[26] Ueki M, Mies G, Hossmann KA. Effect of alpha-chloralose halothane pentobarbital and nitrous oxide anesthesia on metabolic coupling in somatosensory cortex of rat. Acta Anaesthesiol Scand 1992;36:318-22.

[27] Tsekos NV, Zhang F, Merkle H, Nagayama M, Iadecola C, Kim SG. Quantitative measurements of cerebral blood flow in rats using the FAIR technique: correlation with previous iodoantipyrine autoradiographic studies. Magn Reson Med 1998;39:564-73.

[28] Liu ZM, Schmidt KF, Sicard KM, Duong TQ. Imaging oxygen consumption in forepaw somatosensory stimulation in rats under isoflurane anesthesia. Magn Reson Med 2004;52:277-85.

[29] Wu G, Luo F, Li Z, Zhao X, Li SJ. Transient relationships among BOLD CBV and CBF changes in rat brain as detected by functional MRI. Magn Reson Med 2002;48:987-93.

[30] Shen Q, Ren H, Duong TQ. CBF BOLD CBV and CMRO(2) fMRI signal temporal dynamics at 500-msec resolution. J Magn Reson Imaging 2008;27:599-606.

[31] Mégevand P, Quairiaux C, Lascano AM, Kiss JZ, Michel CM. A mouse model for studying large-scale neuronal networks using EEG mapping techniques. Neuroimage 2008;42:591-602.

[32] Paxinos G, Watson C. The rat brain in stereotaxic. Coordinates. San Diego: Academic Press; 1998

[33] Diamond ME, von Heimedahl M, Knutsen PM, Kleinfeld D, Ahissar E. Where snd what in the whisker sensorimotor system. Nat Rev Neurosci 2008;9:601-12.

[34] Zhao F, Zhao T, Zhou L, Wu Q, Hu X. BOLD study of stimulation induced neural activity and resting state connectivity in metomidinesedated rats. Neuroimage 2008;39:248-60.

[35] Huttunen J, Gröhn O, Penttonen M. Coupling between simultaneously recorded BOLD response and neuronal activity in the rat somatosensory cortex. Neuroimage 2008;39:775-85.

[36] Sanganahalli BG, Herman P, Hyder F. Frequency-dependent tactile responses in rat brain measured by functional MRI. NMR Biomed 2008;21:410-6.

[37] Ureshi M, Matsuura T, Kanno I. Stimulus frequency dependence of the linear relationship between local cereabral blood flow and field potential evoked by activation of the rat somatosensory cortex. Neurosci Res 2004;48:147-53.
[38] Hewson-Stoate N, Jomes M, Martindale J, Berwick J, Mayhew J. Further nonlinearities in neurovascular coupling in rodent barrel cortex. Neuroimage 2005;24:535-74.

[39] Martin C, Martindale C, Berwick J, Mayhew J. Investigating neuralhemodynamic coupling and the hemodynamic response function in the awake rat. Neuroimage 2006;32:33-48.

[40] Lindauer U, Villringer A, Dirnagl U. Characterization of CBF response to somatosensory stimulation: model and Influence of anesthetics. Am J Physiol 1993;264:H1223-8.

[41] Nakao Y, Itoh Y, Kuang T, Cook M, Jehle J, Sokoloff L. Effect of anesthesia on functional activation of cerebral blood flow and metabolism. Proc Natl Acad Sci U S A 2001;98:7593-8.

[42] Canals S, Beyerkein M, Murayama Y, Logothetis NK. Electric stimulation fMRI of the perforant pathway to the rat hippocampus. Magn Reson Imaging 2008;26:978-86.

[43] Austin VC, Blamire AM, Allers KA, Sharp T, Styles P, Matthews PM, et al. Confounding effects of anesthesia on functional activation in rodent brain: a study of halothane and alpha-chloralose anesthesia. Neuroimage 2005;24:92-100.

[44] Mathiesen C, Caesar K, Akgoren N, Lauritzen M. Modification of activity-dependent increases in cerebral blood flow by excitatory synaptic activity and spikes in rat cerebellar cortex. J Physiol 1998;512:555-66.

[45] Logothetis NK, Guggenberger H, Peled S, Pauls J. Functional imaging of the monkey brain. Nat Neurosci 1999;2:555-62.

[46] Logothetis NK, Pauls J, Augath M, Trinath T, Oeltermann A. Neurophysiological investigation of the basis of the fMRI signal. Nature 2001;412:150-7.

[47] Rees G, Friston K, Koch C. A direct quantitative relationship between the functional properties of human and macaque V5. Nat Neurosci 2000;3:716-23.

[48] Logothetis NK. The underpinnings of the BOLD functional magnetic resonance imaging signal. J Neurosci 2003;23:3963-71.

[49] Sheth SA, Nemoto M, Guiou M, Walker M, Pouratian N, Toga AW. Linear and non-linear neuronal activity oxygen metabolism and hemodynamic responses. Neuron 2004;42:347-55.

[50] Buxton RB. The elusive initial dip. Neuroimage 2001;13:953-8.

[51] Boxerman JL, Bandettini PA, Kwong KK, Baker JR, Davis TL, Rosen $\mathrm{BR}$, et al. The intravascular contribution to fMRI signal change: Monte-Carlo modeling and diffusion-weighted studies in vivo. Magn Reson Med 1995;34:4-10.

[52] Buxton RB, Uludag K, Dubowitz DJ, Liu TT. Modeling the hemodynamic response to brain activation. Neuroimage 2004;23: S220-33. 